Internat. J. Math. \& Math. Sci.

Vol. 22, No. 4 (1999) 667-688

S 0161-1712<99>22667-1

(C) Electronic Publishing House

\title{
SIR JAMES LIGHTHILL AND MODERN FLUID MECHANICS A MEMORIAL TRIBUTE
}

\section{LOKENATH DEBNATH}

(Received 15 December 1998)

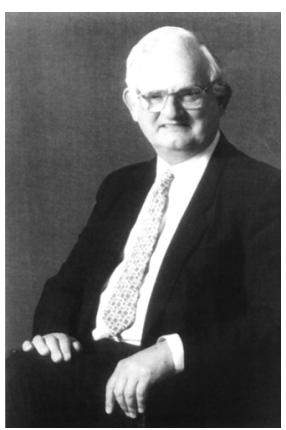

“... as Sir Cyril Hinshelwood has observed ... fluid dynamicists were divided into hydraulic engineers who observed things that could not be explained and mathematicians who explained things that could not be observed."

James Lighthill

Sir James Lighthill

(1924-1998)

ABSTRACT. This memorial tribute commemorates Sir James Lighthill the man and the mathematical scientist. A brief biographical information is presented with my personal reminiscences. His major contributions to fluid mechanics with special reference to nonlinear dispersive waves are discussed. By any standards, his legacy of research and publications of six books and one hundred fifty papers will remain forever as a remarkable example of scholarly work.

Keywords and phrases. Fluid dynamics, nonlinear dispersive waves, aerodynamics, biofluid mechanics.

1991 Mathematics Subject Classification. 01A70, 76B15, $76 Z$.

1. Brief biographical sketch and career. Sir James Lighthill was born on January 23, 1924 in Paris, France. After a rigorous education at Winchester College with an outstanding record, he entered the Trinity College, Cambridge in 1941 to pursue a B.A. degree in Mathematics. He successfully completed his B.A. degree in two years, and then joined British National Physical Laboratory (NPL) as Junior Scientific Officer at Aerodynamics Division in 1943, and soon became a Senior Scientific Officer at NPL. Before he became twenty in 1944, Sir James published his first paper on two-dimensional supersonic airfoil theory in the Aeronautical Research Committee Report and Memorandum (ARCRM). He continued his research at NPL and then published several other papers on the supersonic theory of airfoil in ARCRM in 1944 and 1945. Because of his outstanding research work at NPL, he was awarded a prestigious Research Fellowship 
at Trinity College in 1945. After spending a year at his 'Alma Mater', Lighthill joined the University of Manchester as Senior Lecturer in Mathematics at the age of twenty two. Within a short period of time, he succeeded Sydney Goldstein in the Beyer Professor of Applied Mathematics at Manchester at the age of twenty six. This was almost an extraordinary accomplishment in the United Kingdom in those days. His thirteen years stay at Manchester was very productive and rewarding in his life in terms of research, publications and supervision of doctoral students-many of them are now well known in applied mathematics. Highly respected by his applied mathematics peers, he was instrumental in organizing a strong research group in fluid mechanics and applied mathematics in England. Above all, he created an enduring cordial atmosphere of mutual support and encouragement for fluid dynamics research at the highest level.

In 1952, Lighthill was elected Fellow of Royal Society of London at the age of twenty nine for his pioneering research on sound generated aerodynamically. He was awarded the Bronze Medal of the Royal Aeronautical Society for his outstanding contributions to aerodynamics in 1955.

Lighthill left Manchester in 1959 to take the extremely prestigious position of Director of the Royal Aircraft Establishment (RAE) at Farnborough. His main leadership role included managing the work of the fourteen hundred scientists and engineers, and the eight thousand RAE staff. Towards the end of his five year stay at RAE, he became dissatisfied due to lack of support for applied mathematics from national agencies. However, even as Director of RAE, he was actively involved in research and development of aerodynamics of the slender delta wing for aircraft, spacecraft, and performance of high speed jet aircraft and missiles.

In 1964, Sir James was selected for the position of Royal Society Research Professor at the Imperial College of Science and Technology at London. In a new and conducive academic atmosphere at Imperial College, he had the usual responsibility of teaching, research and guiding many promising young researchers. As his career progressed he was noted for changing his research from one area to another. At Imperial College, he delivered three courses of lectures on Nonlinear Waves, Geophysical Fluid Dynamics and Blood Flow in Arteries during 1965-1967. In addition to his many outstanding papers and presentations on these topics, he got involved in the development of strong research in mathematical biofluid dynamics. Under his unique leadership, a new Institute of Mathematics and Its Applications was established in the United Kingdom in 1965, and he became the first President of this newly created institute. During 19631965, he was awarded Royal Society Medal, Gold Medal from the Royal Aeronautical Society, and Timoshenko Medal from the American Society of Mechanical Engineers for his outstanding research contributions.

From Imperial College, Lighthill returned to Cambridge in 1969 to succeed Paul Dirac as the Lucasian Professor of Applied Mathematics. This is probably the most prestigious Mathematics Chair in the United Kingdom as this Chair was formerly held by Sir Issac Newton. Lighthill was justifiably proud to tell people that his predecessor in the Lucasian Chair was Newton. Within the next two years of his stay in Cambridge, Queen Elizabeth presented him a Knighthood as Sir James Lighthill. Among his many other honors were Bakerian Lecture in 1961, the 48th Wilbur Wright Memorial Lecture 
in 1960, Wright Brothers Lectures in the United States in 1962, The Symons Memorial Lecture in 1969, John von Neumann Lecture in the United States in 1975, American Institute of Aeronautics and Astronautics (AIAA) Aeroacoustics Award in 1976. During his stay in Cambridge from 1969 to 1979, Sir James vigorously continued his teaching and research on acoustics, more and more wave propagation, geophysical fluid dynamics, biofluid mechanics, ocean and atmospheric dynamics with special reference to prediction of monsoons and tropical cyclones.

In 1979, Sir James left Cambridge to serve in the position of Provost of the University College London (UCL). Many people were pleased with his appointment and expressed their feelings by saying that UCL got a Provost who has academic clout, visibility, and tremendous influence in government, industry and society. Due to his enormous wisdom and communication skills, and strength of character, he remained in that administrative position until his formal retirement in 1989. During this period, he was heavily involved in new developments of the College, in promotion of biological and biotechnological research, fund-raising activities, and in significant improvement of women's appointments in senior positions. Even though he was very busy with administrative duties and responsibilities, Lighthill still maintained his scientific research in areas such as extraction of wave energy, dynamical systems, and biomechanics of the human auditory system. From my personal conversation with him and his wife, Nancy in October 1995, I was delighted to know that Sir James made many significant contributions to the University College London in many different ways.

While he was Provost, he was very active in the presentation of special lectures and in research and national educational affairs. His professional activities included service as President of the International Union of Theoretical and Applied Mechanics, as Member of the Advisory Board for the Research Council, 1980-1986, as Member of Natural Environment Research Council and Chairman of its Oceanography and Fisheries Research Committee, 1965-1970, as Member of Geddes Committee of Inquiry into Shipbuilding Industry, President of the International Commission on Mathematical Instruction, 1971-1975, and as Member of the Advisory Council on Research and Development, 1978-1981. As President of ICMI which was then a sub-commission of the International Mathematical Union (IMU), Lighthill has had a great influence on mathematics education at the international level. In order to promote mathematics teaching (Lighthill, 1970) at all levels and to upgrade mathematics education all over the world, he helped organize a number of symposia that include 'New topics in applicable mathematics in Secondary Schools' in Luxembourg in 1973, and 'Mathematics and Language' in Kenya in 1974. His philosophy of mathematics education can be best described by citing his own quotation from his Presidential address at Exeter in 1973. "Let's go beyond mere use of the concrete example as an aid to understanding or of reference to utility as an aid to widening the circle of those in whom interest is aroused. There is a still more important prize to be won: a prize concerned with a deeper integration of mathematics into the total education of the individual.

"I want to suggest that educators may have most benefited their pupils when they have succeeded in giving them a feel for what is involved in the process of applying mathematics... Computers may be of great value in problem-solving, but apparently 
the human brain alone is able to tackle the subtler aspects of creating an effective correspondence between the mathematical world and the world of experiment and observation."

In addition, Sir James delivered many special lectures including the Inaugural Frederic Constable Lecture in 1980, and the Rayleigh Lecture in the United States in 1989. No doubt, he has made some important contributions to the British higher educational system and science policy. Among his many degrees, honors and awards, he received a Gold Medal from the Institute of Mathematics and Its Applications in 1982, and the Harvey Prize of the Israel Institute of Technology in 1981. He also received twenty four honorary degrees from many different universities including Princeton, Brown, and Kiev. In recognition of his notable research contributions to fluid mechanics and applied mathematics, he was elected to twelve learned societies including the US National Academy of Sciences, Russian Academy of Sciences, and the Indian National Science Academy.

After his formal retirement from the University College London in 1989, he was appointed Emeritus Scientist by the College. He continued his research, publications and presentations. He accepted the Royal Society Lectureship to deliver the Humphry Davy Lecture in 1991 and the Inaugural Perkins Memorial Lecture in 1995. In the same year, Sir James and Lady Nancy visited the University of Central Florida for a week during October to deliver three lectures on fluid mechanics as part of the Distinguished Lecture Series in Mathematics at UCF. In 1993, he received the ICASE/LARC Theodorsen Lectureship Award. In November 1996, Sir James and Lady Nancy came to Florida State University at Tallahassee to attend the International Symposium on Theoretical and Computational Fluid Mechanics which was organized in honor of Sir James Lighthill to celebrate his monumental contributions to fluid mechanics, applied mathematics, and the mathematical community of the world. After the symposium, they returned to England. Sir James remained at the University College London until his death on July 17, 1998 after completing a nine-hour swim around the island of Sark against high tides and strong winds. The 1998 Copley Medal of the Royal Society (the highest award of the Royal Society) has been awarded posthumously to Sir James.

2. Personal reminiscences. Perhaps it is appropriate to record here my personal relationship and association with Sir James Lighthill. Before I came to England, I heard his name as one of the most prominent applied mathematicians, and read his famous book Introduction to Fourier Analysis and Generalized Functions and some research papers in order to prepare myself for pursuing research in fluid dynamics at Imperial College London. After joining the Imperial College as a postgraduate student, I first came into contact with him in the Fall of 1965. During the next two years of my stay at Imperial College, I had the rare opportunity of attending his three courses of lectures on nonlinear waves, geophysical fluid dynamics, and blood flow in arteries. All of his three courses of lectures were primarily based on his own research notes and research papers either already published or under preparation at that time in Imperial College. All of these lectures dealt with remarkably new mathematical and physical ideas, results and predictions. I thoroughly enjoyed and learned a great deal from his lectures, and immediately decided to work on linear and nonlinear water waves for my Ph.D. 
dissertation. Subsequently, I wrote my Ph.D. dissertation on Transient Wave Motions in Fluids and successfully finished my degree. Sir James' unlimited enthusiasm, great challenge and interest in teaching and research will remain a lifelong memory with me and my other classmates at Imperial College. As I had stated earlier, his influence on me can, in fact, be traced back to my postgraduate days at the University of Calcutta well before I first met with him at Imperial College.

Probably, it is not out of place to mention my experience in Imperial College and then at the University of Cambridge. During my stay at Imperial from 1965 to 1967, I attended many other courses of lectures in both mathematics and mathematical physics given by many professors. They included Professors J. W. Dungey, W. K. Hayman, W. A. Hepner, H. Jones (then Head of Mathematics), F. G. Leppington, D. W. Moore, S. Rosenblat, Abdus Salam, R. S. Scorer, R. F. Streater, J. T. Stuart, F. W. G. Warren and Ffowcs Williams. In addition, I went to the University College to attend two courses of seminars given by Professor K. Stewartson. During my one year stay in Cambridge as a postdoctoral research fellow, I attended courses of lectures given by Professors P. M. Dirac, T. Brooke Benjamin, and still I vividly remember Lighthill's most interesting and stimulating lectures and his lecture notes prepared for postgraduate students and faculty members. Also what I do remember is the excitement they generated, but most importantly his numerous examples of applications intended to deepen our understanding of the mathematical theory with physical insight. Sir James was an extremely dynamic and inspiring teacher in the classroom. Indeed, he was my most favorite teacher in my student days in London and Cambridge.

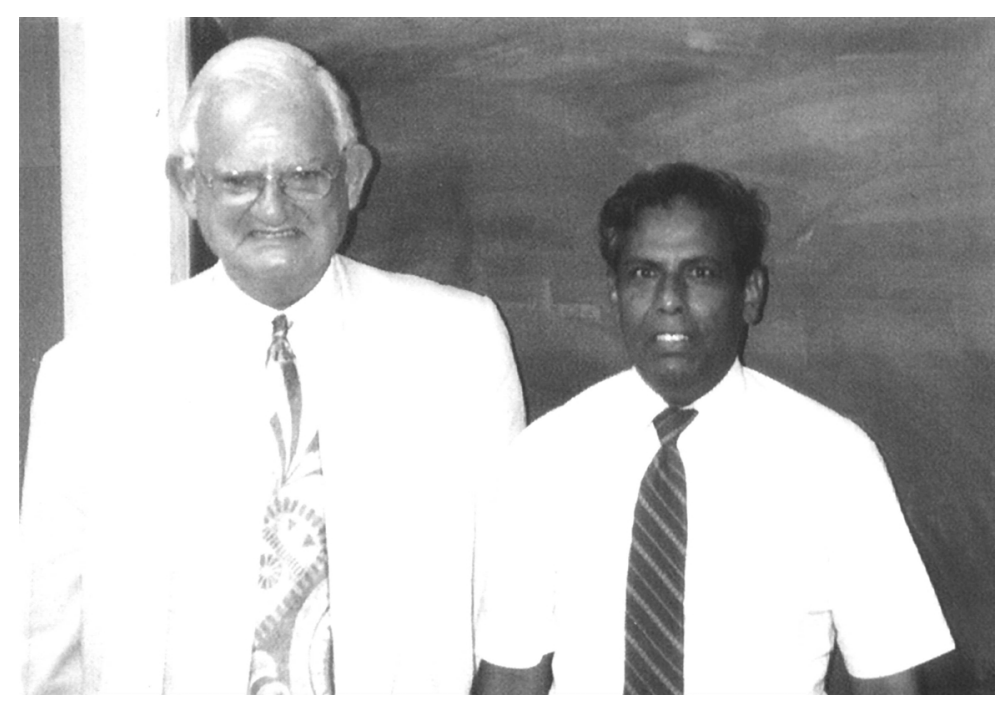

At the University of Central Florida, October 1995. Left to right: Sir James Lighthill and Lokenath Debnath.

Based on my research work, I first gave a seminar on Transient Development of Water Waves at Imperial College in the Spring of 1966. In my talk, I pointed out an 
error in a paper by J. W. Miles on the asymptotic solution of an initial value problem in water waves, and tried to obtain correct asymptotic solution with right physical interpretation. The audience at my seminar consisted of many postgraduate students and faculty members including Professors Lighthill and Scorer. At the end of my presentation, Professor Scorer raised a doubt in my asymptotic analysis, and then also in asymptotic methods that I had used to solve the problem. He was almost sure that the transient component of the asymptotic solution should decay to zero in the limit as time $t \rightarrow \infty$ so that the steady state solution can be obtained. In an immediate response to Scorer's comments, Professor Lighthill said that Debnath's claim seemed to be correct. The transient term obtained by Debnath does not decay to zero in the limit as $t \rightarrow \infty$, possibly due to the Dirac delta function disturbance used as a wave generating mechanism. Obviously, I was delighted to hear the comments of Professor Lighthill in support of my claim. At the request of Lighthill, one of his postgraduate students, M. S. Howe borrowed my research notes and repeated the calculation in order to detect any error in my asymptotic analysis. Fortunately, no error was found. Mr. Howe and I discussed my research problem with Professor Lighthill. Interestingly enough, Lighthill was impressed with my work and then gave a nice physical interpretation of my solution.

During my stay at Imperial College, I had many opportunities to discuss my research problems with Sir James Lighthill and other members of his group which included several British and Indian scholars including Professor P. L. Bhatnagar, Drs. C. Devanathan and A. C. Srivastava. I also attended two annual meetings of the British Theoretical and Applied Mechanics Association at Southampton in 1966 and at Oxford in 1967. In both of these meetings, Sir James Lighthill was a keynote speaker. I enjoyed his outstanding lectures on rotating fluids and on nonlinear waves.

As I have indicated before, Sir James was Lucasian Professor of Applied Mathematics in Cambridge from 1969 to 1979, and then became Provost of the University College London from 1979 to 1989. During this period of twenty years, I visited Cambridge for a year and then Oxford for six months to conduct research in applied mathematics. When I was at the Department of Applied Mathematics and Theoretical Physics in Cambridge, I again had the opportunity to attend some of his lectures and discuss research problems with him. During my stay in Oxford in 1980, I went to the University College London to discuss my research problems on nonlinear diffraction of water waves based on his famous 1979 paper on "Waves and Hydrodynamic Loading." Subsequently, I wrote a paper on "A Theory of Nonlinear Waveloading on Offshore Structures" in 1981, and then a chapter on "Nonlinear Diffraction of Water Waves" for my book entitled Nonlinear Water Waves published in 1994. Meetings with him, which I would look forward to with pleasure, were relaxed-even when dealing with difficult mathematical problems. Despite his very busy schedule, Sir James still was willing to discuss research problems with his students and colleagues with interest and enthusiasm. His kind, warm and dynamic personality was appreciated by students, colleagues, and friends. He was also noted for his leisure interests in swimming, languages, and music throughout his life.

During my tenure as Chair of Mathematics at the University of Central Florida, I established a Distinguished Lecture Series in 1983. Many Nobel Prize winners, Field Prize 
winners, members of the National Academy of Sciences and Fellows of the Royal Society participated in this Lecture Series for a period of fourteen years. At my personal invitation, Sir James Lighthill and Lady Nancy Lighthill visited our department in the Fall of 1995. During this week long visit at Central Florida, he delivered three lectures on hurricane dynamics, bacterial biofluid dynamics, and some aspects of aeroacoustic of extreme-speed jets. As usual, these lectures were loaded with many new, important and interesting ideas and results, and have stimulated interest in applied mathematical research. During this visit to Orlando, we had the opportunity to discuss many issues of mutual interest including research topics, the future of applied mathematics research, education and training in the UK and in the USA. One day, I escorted both James and Nancy on a sightseeing tour at Walt Disney World in Orlando and it seemed to me they enjoyed the tour. However, I enjoyed their company for a week and was impressed with their high levels of thinking with grace, good humor and friendliness. We had an ample but great time and this time we became good friends and were free to discuss many topics of diverse interests including past stories of our stay at London, Cambridge, and Oxford. In response to one of my questions about publication of the second edition of his famous and delightful book on Fourier Analysis and Generalized Functions that was first published in 1958, Lighthill said that forty thousand copies of this book were sold so far. He has neither plan nor intention of making its second edition. This short book is a classic in which he developed the theory of Fourier transforms, generalized functions and asymptotic analysis of Fourier transforms accessible to everyone.

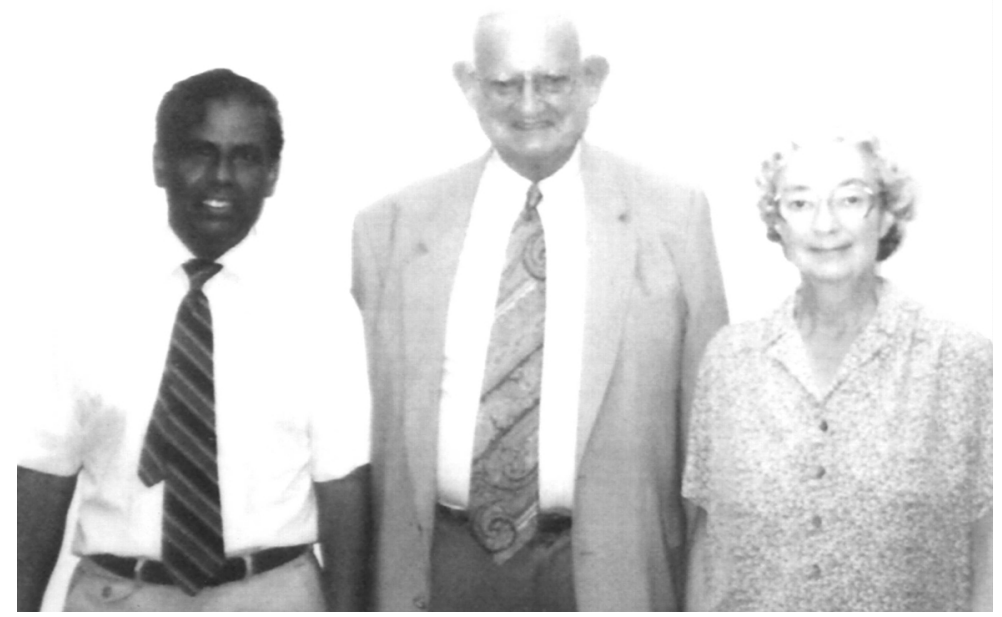

At the University of Central Florida, October 1995.

Left to right: Lokenath Debnath, Sir James Lighthill and Lady Nancy Lighthill

After their return to London, I have had regular correspondences with him. In each of his many letters written to me, he signed his first name in the end. It is my pleasure to display one such letter here to record his friendliness and kind invitation to participate 
in the International Symposium organized in honor of Sir James at Tallahassee, Florida in November 1996. Unfortunately, I missed this unique opportunity of attending this symposium to pay a special tribute to my favorite professor and friend due to my prior commitment to visit India as Fulbright Professor from July to December of 1996. After my return from India, I resumed correspondences with him and was delighted to see his Collected Works in four volumes edited by Professor M. Yousuff Hussaini and published by Oxford University Press. My last letter to him was in late June or early July in 1998 with a special proposal for publishing a book entitled Sir James Lighthill. Unfortunately, I did not receive any response to this letter, possibly due to his sudden death in July. I was deeply disturbed by this shocking news of his death. There is absolutely no doubt that this is a great loss for the entire mathematical as well as scientific community of the world. Personally, I lost a great teacher, and a great friend to whom I would remain grateful for his help and encouragement in my life. Indeed, I was fortunate to come in contact with such a great applied mathematician in both personal and professional levels. So my desire as well as interest in writing this memorial tribute commemorating Sir James Lighthill the man and the mathematical scientist is founded solely on my deep respect and admiration for this great man and renowned applied mathematician whom I had the opportunity of knowing very well.

3. Significant research contributions. Even a glance at the chronological list of his research papers and books appended to Collected Papers of Sir James Lighthill (1997) will show that his whole life was packed with research and publication activities. His creativity was simply phenomenal, and his deep involvement in teaching, academic and professional service was enormous, and has many ramifications. I have no intention of discussing all of his contributions in many areas which include aerodynamics, fluid dynamics, waves in fluids, biofluid mechanics, geophysical fluid dynamics, boundary layer theory, magnetohydrodynamics, atmospheric and ocean dynamics. In each of these areas, he made major and unique contributions with new mathematical results and profound physical significance. His mathematical research always dealt with vivid imagination that responded to the 1983 quotation of V. I. Arnold: "In every mathematical investigation, the question will arise whether we can apply our mathematical results to the real world."

Sir James Lighthill's research career began at the National Physical Laboratory with publications in ARCRM during 1943-1945. His pioneering work on sound generated aerodynamically appeared in the Proceedings of the Royal Society of London in 1952. This work along with his many other papers on high-speed aerodynamics, how flows generate sound, and the theory of jet noise are considered as classic, and led to a new branch of fluid mechanics which is called aeroacoustics, and has immense industrial and technological importance. Indeed, he is considered as the founder of the modern theory and applications of aeroacoustics that basically deals with the mutual interaction of sound with fluid flows and turbulence as a source of sound. He was probably the first applied mathematician who developed the whole of modern aeroacoustics from unified mathematical and physical viewpoints. In his famous 1952 papers, Sir James gave an entirely new and novel derivation of a formula for the sound radiation field in the form 


$$
\rho-\rho_{0} \sim \frac{1}{4 \pi a_{0}^{2}} \int \frac{\left(x_{i}-y_{i}\right)\left(x_{j}-y_{j}\right)}{|\mathbf{x}-\mathbf{y}|^{3}} \frac{i}{a_{0}^{2}} \frac{\partial^{2}}{\partial t^{2}} T_{i j}\left(\mathbf{y}, t-\frac{|\mathbf{x}-\mathbf{y}|}{a_{0}}\right) d y .
$$

This is now known as the Lighthill formula that gives an exact estimate for the total energy radiated and its directional distribution. This led him to formulate a new fourth power law for the density variations (3.1) which is proportional to the product

$$
\frac{1}{a_{0}^{2}} \frac{1}{x} \frac{1}{a_{0}^{2}}\left(\frac{U}{l}\right)^{2} \rho_{0} U^{2} l^{3}=\rho_{0}\left(\frac{U}{a_{0}}\right)^{4} \cdot\left(\frac{l}{x}\right) .
$$

This formula gives a remarkle description of the dependence of the density changes in the sound radiation field on the fourth power of the Mach number $M=\left(U / a_{0}\right)$. He then derived the sound intensity which is roughly proportional to $\left(a_{0}^{3} / \rho_{0}\right)$ times the square of (3.2). In other words, Lighthill proved the formula

$$
I(x) \sim \rho_{0}\left(\frac{U^{8}}{a_{0}^{5}}\right)\left(\frac{l}{x}\right)^{2} .
$$

Thus, he established a precise formula for the total acoustic power output which is roughly proportional to

$$
\rho_{0} U^{8} a_{0}^{-5} l^{2}
$$

This is the famous Lighthill eighth power law which has had almost simultaneous experimental confirmation. He then extended his theory of sound generated aerodynamically by taking into account the statistical properties of turbulent airflows, from which the sound radiated is called aerodynamic noise. His remarkable physical explanation of the theory of aerodynamic noise generation was simultaneously verified by several experimental works on both subsonic and supersonic jets. His mathematical work was so fundamental that it remains today the only practical means for computing noise in complex flow configurations. So his deep and intricate research on aeroacoustics influenced all subsequent work on new aircraft engine designs in the second half of the twentieth century. In the early nineties, Lighthill delivered many special lectures on different aspects of the aeroacoustics of high-speed jets by describing a new dynamical theory of nonlinear propagation of statistically random assemblages of conical shock waves which are radiated by eddies convected at supersonic speed. He showed, by both a general theoretical analysis and by computational method, how their propagation is dominated by a characteristic "bunching process"-a name given by Lighthill himself. This marked process is associated with a strong tendency of early local unions of shock waves that act to stimulate further unions with neighboring shock waves. His completely general theoretical study led to the demonstration of the universal tendency towards bunching process.

In his 1956 famous paper "On Viscosity Effects in Sound Waves of Finite Amplitude" written in honor of the seventieth birthday of Sir G. I. Taylor, Lighthill initiated the theory of nonlinear acoustics. As a follow-up of this work, he developed mathematical theory of gas dynamics interacting with gas physics. He then published two major papers "Dynamics of a Dissociating Gas, Part 1" and "Equilibrium Flow, Part 2, Quasiequilibrium Transfer Theory" in the Journal of Fluid Mechanics in 1957. His dealt with 


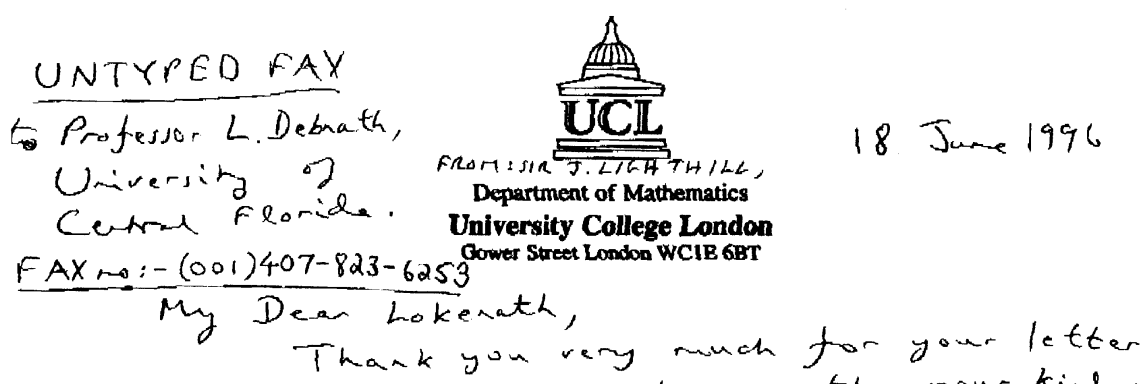
of 11 June. I apraciate greatly jour kindaess in inviting he for a return risit to UCF. in inviting he for a reture the won't be possible, Profersor Hassaimi (the former ICASE Diecher, mow te FSU) includes both (i) the uterrational meeting in FSU (mor 6 to 8 Nuvenber) to rark the publication of ray collected pintific paper in 4 volumes, followed immadiately by (ii) on tha (smaller) meetis at ICASE. It is, I'r a traid, Mrmply vilte. iryosinbe for we bo fir in a new Orlanks vilt. Personally, I would wost kealy watcome it, Lokerath, if you should in practice be able to be a partid be delizpal ho mecting in itakion for you If thes would alp If Is there any chance of that?

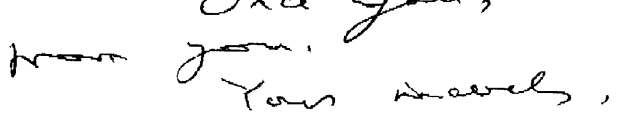

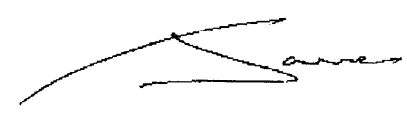


predictions on the velocity field originated from acoustic noise and a generalized turbulence in a layer overlaying a convectively unstable atmospheric region.

In the area of linear and nonlinear waves in fluids, Lighthill made many significant contributions during 1954-1989. I have been familiar with almost all of his contributions to many different problems of generation and propagation of waves in fluids. In collaboration with T. B. Benjamin, Lighthill's first important work on cnoidal waves and bores was published in the Proceedings of the Royal Society, London in 1954. He also published two joint papers (1955) with G. B. Whitham, "On Kinematic Waves, I and II" which deal with a new class of waves different from the classical dynamic wave motions described by Newton's second law of motion. They developed the theory and applications of kinematic waves to several real-world problems which include flood waves in rivers, glacier flow, chromatographic models, and traffic flows on highways. This work has had widespread applications to other areas of physics and chemistry. In 1962, Lighthill wrote another major paper on "Physical Interpretation of the Mathematical Theory of Wave Generation by Wind." He predicted that travelling water waves in a wind produce, to a first approximation, airflow undulations with pressures minimum over crests and maximum over troughs. He also described the physical mechanism of energy transfer from wind to water waves. The rate of this energy transfer varies as the square of the wave amplitude, and therefore, as the wave energy itself which grows exponentially.

In 1960, Sir James Lighthill's deep and profound paper provided an elegant mathematical theory with physical interpretation of magnetohydrodynamic waves and other anisotropic wave motions. He developed a new method for finding the asymptotic behavior at large distances (as $r \rightarrow \infty$ ) of the solution of an equation of the form

$$
P\left(\frac{\partial^{2}}{\partial t^{2}}, \frac{\partial^{2}}{\partial x^{2}}, \frac{\partial^{2}}{\partial y^{2}}, \frac{\partial^{2}}{\partial z^{2}}\right) u=e^{i \omega t} f(\mathbf{r}),
$$

where $P$ is a polynomial in partial derivatives, $\mathbf{r}=(x, y, z)$ and $f(\mathbf{r})$ is a function with a compact support in $\mathbb{R}^{3}$.

Using the three-dimensional Fourier transform with respect to space variables $x, y$, and $z$, he solved equation (3.5) in the form

$$
u=e^{i \omega t} \int_{-\infty}^{\infty} \int_{-\infty}^{\infty} \int_{-\infty}^{\infty} \exp [i(\boldsymbol{\kappa} \cdot \mathbf{r})] U(\boldsymbol{\kappa}) d \boldsymbol{\kappa}
$$

where $U=F / G, F(\boldsymbol{\kappa})=\mathscr{F}\{f(\mathbf{r})\}$, and

$$
G(\boldsymbol{\kappa})=P\left(-\omega^{2},-k^{2},-l^{2},-m^{2}\right),
$$

and $\boldsymbol{\kappa}=(k, l, m)$. He then obtained the unique solution of physical interest by different ways of applying the Sommerfeld radiation condition. He also expressed the asymptotic behavior of (3.6) as $r \rightarrow \infty$ in terms of the singularities of the integrand. Finally, he solved a more general initial value problem of a more general equation of the form

$$
P\left(\frac{\partial^{2}}{\partial t^{2}}, \frac{\partial^{2}}{\partial x^{2}}, \frac{\partial^{2}}{\partial y^{2}}, \frac{\partial^{2}}{\partial z^{2}}\right) u=f(\mathbf{r}, t)
$$


Application of the four-dimensional Fourier transform with respect to three space variables and time $t$ gives the solution in the form

$$
u(\mathbf{r}, t)=\int_{-\infty}^{\infty} \int_{-\infty}^{\infty} \int_{-\infty}^{\infty} \int_{-\infty}^{\infty} \exp [i(\boldsymbol{\kappa} \cdot \mathbf{r}+\omega t)] \frac{F(\boldsymbol{\kappa}, \omega)}{G(\boldsymbol{\kappa}, \omega)} d \boldsymbol{\kappa} d \omega .
$$

Thus, he obtained a solution for $u$ which is zero for $t<0$, and there is no complex roots of $\omega$ for which $G=0$ with real $k, l$, and $m$. Consequently, the resulting solution becomes

$$
u(\mathbf{r}, t)=\int_{-\infty}^{\infty} \int_{-\infty}^{\infty} \int_{-\infty}^{\infty} \int_{-i \epsilon-\infty}^{-i \epsilon+\infty} \exp [i(\boldsymbol{\kappa} \cdot \mathbf{r}+\omega t)] \frac{F}{G} d \boldsymbol{\kappa} d \omega
$$

Then the asymptotic representation of (3.10) is obtained as $r \rightarrow \infty$ and $t \rightarrow \infty$ simultaneously.

In his 1964 inaugural lecture as the first President of the Institute of Mathematics and Its Applications, Sir James discussed a wide range of different aspects of the theory of group velocity. In this paper, he obtained a new formula of the theory of group velocity in three dimensions. He also developed the method of stationary phase in three dimensions for a problem of forced wave motion due to a steady source of fixed frequency $\omega_{0}$ with a compact support. His inaugural lecture on "Group Velocity" published in the Journal of the Institute of Mathematics and Its Applications in 1965 is concerned with an elaborate review of the linear theory of dispersive wave phenomena based on either the method of superposition of plane wave solutions or the kinematics of surfaces of constant phase. His general analysis led him to unexpected formulas for energy flux in plane periodic waves in a very general nonlinear conservative dynamical system. These are given in terms of the averaged Lagrangian density $\mathscr{L}$, expressed as a function of the frequency $\omega$ and wavenumber $k$. He found the energy flux as $-\omega \mathscr{L}_{k}$, and the energy density as $\omega \mathscr{L}_{\omega}-\mathscr{L}$. Their ratio representing the group velocity can be expressed as $(d \omega / d k)$ for changes keeping constant the measure of wave amplitude $(\mathscr{L} / \omega)$. He then extended the linear wave theory in another deep and profound paper entitled "On Waves Generated in Dispersive Systems to Travelling Forcing Effects with Applications to the Dynamics of Rotating Fluids" published in JFM in 1967. His general problem of the propagation of dispersive waves generated by travelling forcing effects, that may be steady, oscillatory or transient in nature, is governed by a linear partial differential equation

$$
P\left(i \frac{\partial}{\partial t},-i \frac{\partial}{\partial x},-i \frac{\partial}{\partial y},-i \frac{\partial}{\partial z}\right) \phi=e^{-i \omega_{0} t} f(\mathbf{r}-\mathbf{U} t),
$$

where $P$ is a polynomial, $\phi(x, y, z, t)$ is the disturbance, and the forcing term $f$ vanishes outside a finite region around the origin. Using the three dimensional Fourier transform with respect to $x, y$, and $z$, he obtained the general formal solution

$$
\phi(\mathbf{r}, t)=\int_{-\infty}^{\infty} \int_{-\infty}^{\infty} \int_{-\infty}^{\infty} \frac{F(\boldsymbol{\kappa}) \exp \left[i\left\{-\omega_{0} t+\boldsymbol{\kappa} \cdot(\mathbf{r}-\mathbf{U} t)\right\}\right]}{P\left(\omega_{0}+\mathbf{U} \cdot \boldsymbol{\kappa}, \boldsymbol{\kappa}\right)} d \boldsymbol{\kappa}
$$

where $\boldsymbol{\kappa}=(k, l, m)$ is the wavenumber vector, and $F(\boldsymbol{\kappa})$ is the Fourier transform of $f(\mathbf{r})$. He then used his own asymptotic method (1960) of evaluation of integrals such 
as (3.12) at distances from the forcing region large compared with the dimension. The asymptotic value of (3.12), as defined by replacing $\omega_{0}$ by $\omega_{0}+i \epsilon$ in (3.12) and making $\epsilon \rightarrow 0$ is determined. In the wavenumber space $\boldsymbol{\kappa}=(k, l, m)$, at each point of the surface

$$
P\left(\omega_{0}+\mathbf{U} \cdot \boldsymbol{\kappa}, \boldsymbol{\kappa}\right)=0
$$

on which the denominator of (3.12) vanishes, an arrow is drawn normal to the surface, selecting from the two normal directions the one pointing towards the surface $P\left(\omega_{0}+\right.$ $\mathbf{U} \cdot \boldsymbol{\kappa}+\delta, \boldsymbol{\kappa})=0$, where $\delta$ is small and positive. Then, waves are determined in any particular direction stretching out from the forcing region with their amplitude given asymptotically by the formula

$$
\frac{4 \pi^{2}}{|K|^{\frac{1}{2}} R} \cdot \frac{F(\boldsymbol{\kappa})}{\left|\nabla_{\boldsymbol{\kappa}} P\left(\omega_{0}+\mathbf{U} \cdot \boldsymbol{\kappa}, \boldsymbol{\kappa}\right)\right|},
$$

where $R=|\mathbf{r}-\mathbf{U} t|$ represents distance from the forcing region, and $K$ is the Gaussian curvature (product of principal curvatures) of the wavenumber surface (3.13). The waves whose frequency is $\omega_{0}$ relative to a forcing region moving at velocity $\mathbf{U}$ must have absolute frequency $\omega_{0}+\mathbf{U} \cdot \boldsymbol{\kappa}$ (the Dopper effect), and hence their wavenumber vector must lie on the surface (3.13). Moreover, the group velocity for waves satisfying the dispersion relation $P(\omega, \boldsymbol{\kappa})=0$ is given by

$$
\left(\frac{\partial \omega}{\partial k}, \frac{\partial \omega}{\partial l}, \frac{\partial \omega}{\partial m}\right)=-\frac{\nabla P}{\partial P / \partial \omega} .
$$

When $\omega_{0}=0$, the equation of the surface (3.13) becomes $P(\mathbf{U} \cdot \boldsymbol{\kappa}, \boldsymbol{\kappa})=0$ which may be interpreted as a statement that a steady forcing effect can generate only waves whose crests are stationary relative to the velocity of the forcing region.

His mathematical theory as well as physical significance of this paper is so general that he employed his theory to study

(i) Rossby waves in a $\beta$-plane ocean due to travelling steady or travelling transient disturbances,

(ii) the classical Kelvin ship wave problem $\left(\omega_{0}=0\right)$,

(iii) internal gravity waves in a stratified fluid due to a vertically moving steady disturbance, and

(iv) Taylor columns generated in a body of uniformly rotating fluid.

The predicted shape of gravity waves generated in a uniformly stratified fluid is in excellent agreement with experimental findings of Mowbray (1966). In summary, his 1967 remarkable paper led to a unified approach to many types of dispersive waves that can be excited in a non-rotating, rotating and/or stratified fluid including the atmosphere and the oceans.

We next turn our attention to Lighthill's deep and significant contributions to the theory of nonlinear dispersive waves. His work in this area witnessed the statement of Werner Heisenberg: “ ... the progress of physics will to a large extent depend on the progress of nonlinear mathematics, of methods to solve nonlinear equations ... and therefore we can learn by comparing different nonlinear problems."

During his stay at Imperial College, Sir James first published his first paper entitled "Contributions to the Theory of Waves in Nonlinear Dispersive Systems" in the 
Journal of the Institute of Mathematics and Its Applications in 1965. He not only began to work on nonlinear wave propagation, but also he provided outstanding leadership in organizing the first "Discussion Meeting on Nonlinear Theory of Wave Propagation in Dispersive Systems" in May 1966. It is a delight to read his Introductory Remarks at this meeting: " ... a relatively new area of study of the mechanics of waves, an area in which most of the work has been done during the past six years. This work, on dispersive systems, represents the second phase of development of the nonlinear mechanics of waves." As expected, this meeting came to an end with an enormous success. In his Closing Remarks, Sir James states: “ ... Methods apparently of diverse character for analysing the nonlinear development of dispersive waves have been described." Perhaps the most interesting statement in his Closing remarks was: " ... Not only those working in the field, but also those still to be attracted into it by all its interesting potentialities, will find the present compendium of knowledge on the subject uniquely valuable." It is my pleasure to record here his influence on my own research on this new subject and on my direct involvement in organizing two NSF-CBMS research conferences on nonlinear waves in 1982 and 1992 in the United States. Influenced by Lighthill's own Closing remarks: “This has been a most interesting discussion to attend, and will, I believe, in its written version, be a work of marked permanent value ...," I have had the opportunity to publish, as a follow up of the NSF-CBMS Conferences, four edited volumes $(1983-1984,1992)$ on the theory and applications of nonlinear dispersive wave phenomena.

Sir James also wrote a deep and comprehensive paper entitled "Some special cases treated by the Whitham theory" published in the Special Volume A299 (1967) of the Proceedings of the Royal Society. In his two famous papers $(1965,1967)$, Lighthill developed a general theory of nonlinear dispersive waves in conservative systems under conditions when the proportional change in amplitude or wavenumber over a distance of one wavelength is very small. For any dynamical system in this restricted class, a periodic waveform $u=\phi(\theta)$, where $\theta=\omega t-k x$, of a given wavenumber $k$ and amplitude $a$ has a given frequency $\omega=\omega(k, a)$ which may be referred to as nonlinear dispersion relation. When $k$ and $a$ are known, the average Lagrangian $\mathscr{L}(\omega, k, a)$ per unit length can be derived. Then it is possible to eliminate amplitude $a$ from the expression for $\mathscr{L}$ and the nonlinear dispersion relation so that $\mathscr{L}$ can be treated as a function of $\omega$ and $k$ in the form

$$
\mathscr{L}(\omega, k)=\frac{1}{2 \pi} \int_{0}^{2 \pi} L\left(\phi, \phi_{t}, \phi_{x}\right) d \theta,
$$

where $L$ is the Lagrangian of the system.

For a slowly varying wavetrain, the wave field can be described by a phase function $\theta(x, t)$ which is a smoothly varying function and assumes successive integral multiple values of $2 \pi$ on successive wave crests and is now known as the Whitham equation. In terms of $\theta$, the local frequency and local wavenumber are defined by

$$
\omega=-\theta_{t} \quad \text { and } \quad k=\theta_{x} .
$$

With the prescribed phase function $\theta$, Lighthill employed the Whitham averaged variational principle 


$$
\delta \int_{t_{1}}^{t} \int_{x_{1}}^{x_{2}} \mathscr{L}\left(-\theta_{t}, \theta_{x}\right) d x d t=0
$$

This leads to the celebrated Euler equation

$$
\frac{\partial}{\partial t}\left(\frac{\partial \mathscr{L}}{\partial \omega}\right)-\frac{\partial}{\partial x}\left(\frac{\partial \mathscr{L}}{\partial k}\right)=0 .
$$

This is now known as the Whitham fundamental equation for nonlinear dispersion. As pointed out by Whitham (1974), (3.19) is the conservation equation representing the balance between changes in the time-like adiabatic invariant $\mathscr{L}_{\omega}$ and the spacelike adiabatic invariant $\mathscr{L}_{k}$. Other conservation equations can be derived from (3.18) by applying Noether's Theorem. In particular, the invariance of $\mathscr{L}$ with respect to arbitrary time and space translations lead respectively to the energy equations:

$$
\frac{\partial}{\partial t}\left(\omega \mathscr{L}_{\omega}-\mathscr{L}\right)-\frac{\partial}{\partial x}\left(\omega \mathscr{L}_{k}\right)=0
$$

and the momentum equation

$$
\frac{\partial}{\partial t}\left(k \mathscr{L}_{\omega}\right)+\frac{\partial}{\partial x}\left(\mathscr{L} \mathbf{I}-k \mathscr{L}_{k}\right)=0
$$

where the expression $\mathscr{I} \mathbf{I}-k \mathscr{L}_{k}$ is a matrix representing momentum flux.

The definition (3.17) gives another conservation equation

$$
\frac{\partial \omega}{\partial x}+\frac{\partial k}{\partial t}=0
$$

which represents the conservation of wave crests.

Lighthill showed that equation (3.19) can be expressed by means of (3.17) in terms of the phase function $\theta$ as a second-order quasi-linear partial differential equation for $\theta$

$$
\mathscr{L}_{\omega \omega} \theta_{t t}-2 \mathscr{L}_{\omega k} \theta_{t x}+\mathscr{L}_{k k} \theta_{x x}=0,
$$

where all subscripts represent partial derivatives. By an appropriate Legendre transformation, he transformed (3.23) into a linear equation for a new dependent variable $\phi(x, t)=k x-\omega t-\theta, \phi_{\omega}=-t$ and $\phi_{k}=x$ so that $\phi(x, t)$ satisfies the equation

$$
\mathscr{L}_{\omega \omega} \phi_{k k}-2 \mathscr{L}_{\omega k} \phi_{\omega k}+\mathscr{L}_{k k} \phi \omega \omega=0 .
$$

According to the method of characteristics (see Debnath 1967), the characteristics of equation (3.23) represent two distinct families of curves, and satisfy the equation

$$
\mathscr{L}_{\omega \omega}(d \omega)^{2}+2 \mathscr{L}_{\omega k} d \omega d k+\mathscr{L}_{k k}(d k)^{2}=0 .
$$

In both of his papers $(1965,1967)$, Lighthill discussed various aspects of the Whitham theory and established the criterion governing whether dispersion equation (3.19) is hyperbolic or elliptic. He showed that equation (3.25) is hyperbolic or elliptic at a point $(\omega, k)$ if the surface $\mathscr{L}=\mathscr{L}(\omega, k)$ is synclastic or anticlastic. A line separating a synclastic from an anticlastic part of the surface is a transition line on which the equation is parabolic. 
Lighthill obtained the solution of (3.23) as a linear supposition of solutions proportional to $\exp [i(\sigma t-\kappa x)]$, where $\kappa$ is a root of the quadratic equation

$$
\left(\mathscr{L}_{k k}\right)_{0} \kappa^{2}+2\left(\mathscr{L}_{\omega k}\right)_{0} \kappa \sigma+\left(\mathscr{L}_{\omega \omega}\right)_{0} \sigma^{2}=0 .
$$

For the case of deep water waves, his direct calculation reveals that roots of (3.26) are real or complex according to whether the finite amplitude $a>0.054 \lambda$ or $a<0.054 \lambda$. If the roots are complex, the solution of (3.23) can be given by

$$
\theta=\theta_{0}+\epsilon\left(\frac{\sinh \gamma x}{\gamma}\right) \exp \left[i \sigma\left(t-\frac{x}{c_{0}}\right)\right]
$$

where $\theta=\left(k_{0} x-\omega_{0} t\right)$ is the undisturbed value of $\theta$, and $\gamma$ is the rate of exponential growth of modulation with distance given by

$$
\gamma=\frac{\sigma\left[\left(\mathscr{L}_{\omega \omega}\right)_{0}\left(\mathscr{L}_{k k}\right)_{0}-\left(\mathscr{L}_{\omega k}^{2}\right)_{0}\right]^{1 / 2}}{\left(\mathscr{L}_{k k}\right)_{0}},
$$

and $c_{0}$ is the velocity of propagation (group velocity) of the amplitude modulation given by

$$
c_{0}=-\frac{\left(\mathscr{L}_{k k}\right)_{0}}{\left(\mathscr{L}_{\omega k}\right)_{0}} .
$$

When roots of (3.26) are complex, $\gamma$ is real, and hence the amplitude containing the sinh function suggests that the pure gravity waves on deep water become unstable in the sense that slow modulation grows exponentially as the wave propagate in the far field $(x \rightarrow \infty)$.

On the other hand, if $\gamma$ is imaginary, the solution (3.27) is still valid but the interpretation would be entirely different. In contrast to the elliptic case, no exponential growth will occur, but the modulation will propagate at two different velocities $c_{1}$ and $c_{2}$ given by

$$
\frac{\sigma}{c_{1}}=\frac{\sigma}{c_{0}}-|\gamma|, \quad \frac{\sigma}{c_{2}}=\frac{\sigma}{c_{0}}+|\gamma| .
$$

This is the remarkable splitting of the group velocity in the hyperbolic case that was first predicted by Whitham (1967).

The upshot of Lighthill's deep analysis is that, under certain conditions, very gradual variations in the properties of a wavetrain are governed by the elliptic or hyperbolic equations. In the elliptic case, solutions representing the amplitude and the wavenumber for nonlinear dispersive waves definitely become unstable. The most striking feature of the Lighthill theory is that there is a development of wave group whose amplitude distribution is symmetrical about its maximum. This feature is found to remain true at later times, but energy is redistributed within the group so that the maximum amplitude increases with development of a cusp at the center of the group when the wavenumber exhibits a singularity. Moreover, such a singularity occurs after a finite time for a wide class of elliptic initial value problems. Lighthill's theoretical predictions were found in excellent agreement with the theory and the conclusive experimental findings of Benjamin and Feir [22, 23, 24]. 
At Imperial College, Lighthill worked extensively on geophysical fluid dynamics and biofluid dynamics. In 1969, he published his work on "Dynamic Response of the Indian Ocean to Onset of the Southwest Monsoon." Remarkably, his linearized theory of unsteady wind-driven currents in a horizontally stratified ocean with applications to the northern part of the Indian Ocean was fairly general so that it can be applied to other oceanic models. He also discussed barotropic response, baroclinic wave propagation, and baroclinic response to monsoon onset in an equatorial ocean in some detail. Subsequently, he wrote a few articles on monsoon dynamics and made several presentations on hurricane dynamics and tropical cyclones. From his extensive study and research in flood waves, monsoon dynamics, and hurricane dynamics, Lighthill became seriously interested in all kinds of natural hazards that include floods, hurricanes, cyclones, tornadoes, earthquakes, thunderstorms, underwater explosions, landslides, and volcanic eruptions. These hazards are the most common and frequently observed to have catastrophic effects on life, economic, coastal and marine resources. During the last three decades, Sir James showed a tremendous increase in interest in the dynamics of oceans and atmosphere with special reference to waves and currents caused by hurricanes, tropical cyclones, underwater explosions and earthquakes. He became more and more involved in developing protective measures to save life, economic, coastal and marine resources from natural hazards. During the last several years of his life, he served as Chairman of the Special Committee on the International Decade for Natural Disaster Reduction of the International Council of Scientific Unions, and traveled and lectured world-wide. His deep concern and active interest in reduction of all natural hazards and of wasteful use of energy reveal the unique character of this man.

Both the design of wave-energy extraction by submerged resonant ducts and the diffraction of water waves by offshore solid structures are of considerable interest in ocean engineering and naval hydrodynamics. Lighthill's 1979 paper entitled "Twodimensional analysis related to wave-energy extraction by submerged resonant ducts" published in JFM represents his major interest in ocean engineering. This work has many important practical applications for the design of wave-energy extraction devices consistent with the need for maximum seaworthiness. Based on two-dimensional wave hydrodynamics and linearized duct dynamics, he developed both local models of forcing-pressure modification, and fully interactive models. His study led to inferences of many aspects of the duct's response to incident waves including forcing-pressure modification, added mass, and energy absorption, transmission and reflextion.

The study of wave forces on offshore structures or natural boundaries has become more important due to the tremendous growth and need for ocean exploration and extraction of wave energy from oceans. Methods of calculating wave forces on offshore structures and/or harbors based upon Morison's (1950) equation seemed to be useful for construction of such structures that have been widely used for exploration of oil and gas from the ocean floor. However, several difficulties in using it in the design and construction of offshore structures have been reported in the literature. In 1979, Lighthill was invited to deliver a keynote address at the Behavior of Offshore Structures Symposium at Cranfield, England. The title of his keynote address was "Waves and Hydrodynamic Loading." He gave a detailed survey of his title and then 
presented a mathematical analysis of estimating all linear and nonlinear wave forces acting on a very large solid structure of size $b$ comparable with the wavelength $\lambda$, where $\lambda=(2 \pi / k)$. His profound analysis reveals that the nonlinear contributions to the wave loading on offshore structures are made up of the quadratic, dynamic and waterline forces associated with the irrotational flow component of the wave loading. He then suggested a new nonlinear modification of the classical Morison's equation (6.2.5) (see Debnath, 1994) with the nonlinear contribution given by

$$
F_{n l}=F_{q}+F_{d}+F_{w}
$$

where $F_{q}, F_{d}$, and $F_{w}$ are defined by (6.5.13), (6.5.14) and (6.5.16) respectively (see Debnath (1994) p. 294). The second-order correction to the Morison equation for a vertical cylinder is given by

$$
\left(F_{d}+F_{w}\right)=\frac{5}{2}(a k) g \rho a \pi b^{2} \sin \omega t \cos \omega t .
$$

The significance of the nonlinear correction term (3.32) is that it gives an enhanced positive peak of the total drag force, occurring when $\cos \omega t$ and $\sin \omega t$ are both positive. The importance of the correction term increases with the wave steepness $(a k)$. The modified Morison equation (6.2.5) for the case of a vertical cylinder is given by

$$
D=2 g \rho a \pi b^{2} \cos \omega t+\frac{1}{2} C_{D} g \rho a(a b) \sin \omega t|\cos \omega t| .
$$

Finally, the quadratic wave force $F_{q}$ can be expressed in the form

$$
F_{q}=2 \pi g \rho a b^{2}(k b)^{2}(a k)\left[4\left(\log \frac{C}{k b}\right)-\frac{3}{4}\right] \sin 2 \omega t,
$$

where $(k b) \ll 1$. Thus, the quadratic force is insignificant in the limit of very small $(k b)$ and begins to exert its influence as $k b$ increases. The upshot of Lighthill's analysis is that the nonlinear correction is dominated by the sum (3.32) of the dynamic and waterline forces. In the diffraction regime, the quadratic force contributes to the magnitude of those other forces as $k b$ increases. This nonlinear analysis of Lighthill has subsequently extended to the case of fluid of finite depth by several authors including Debnath and Rahman (1981). As a follow-up of his work, Sir James published another paper on "Fundamentals concerning wave loading on offshore structures" in JFM in 1986. This paper was aimed at relating a certain substantial body of established knowledge concerning wave loading on offshore structures to fundamental principles of mechanics of solids and of fluids. In fact, he described some current techniques for calculating wave loading on offshore structures from the standpoint of the fundamental mechanics of solids and fluids.

Sir James began his study and research in biofluid dynamics at Imperial College and continued to work on this subject until his death. I am not fully familiar with all his research papers in this subject, and so, I do not intend to discuss his contributions in this area. However, I find it necessary to mention some of his groundbreaking work on both external biofluid dynamics, the analysis of mechanisms of swimming and flying, and internal biofluid dynamics. The whole volume IV of Collected Papers of Sir James contains all of his fifteen papers on external biofluid dynamics, and five articles on internal biofluid dynamics. The former is concerned with animal locomotion 
through surrounding fluid media (water and air), as animal's flight in air or animal's swimming through water, while the latter deals with fluid motions within an animal as in the study of circulation and respiration. However, these two halves of biofluid dynamics have in common one general feature that distinguishes them from the rest of fluid dynamics: fluid movements are energized by the working of an animal's motile external or internal surfaces, parts of which are highly flexible. Another shared feature of the two areas is their common demand for interdisciplinary study. His major contributions to external biofluid dynamics extend from the first elucidation of the basic mechanisms of aquatic animal propulsion to ciliary and flagellar propulsion, and to animal flight in birds, bats and insects. As a lifelong devotee of fluid mechanics, Lighthill created not only a new major division of biofluid dynamics, but also he raised the subject to a new higher level through his groundbreaking work on both external and internal biofluid dynamics. On one hand, Sir James wrote major papers on aquatic animal locomotion and its hydrodynamics; on the other hand, he published articles on application of hydrodynamical slender-body theory to fish swimming movements. His two papers entitled "Large-amplitude Elongated-body Theory of Fish Locomotion," and "Mathematical Theories of Fish Swimming" represent major contributions to the hydrodynamic interaction between a swimming fish and the water. Even after his retirement, Lighthill continued his research on fish swimming and published a series of papers (1990) on "Balistiform and Gymnotiform Locomotion." More recently, he made fascinating analysis of how a herring can control the motion of its head to enable it to sense pressure differences across the head. He formulated the fundamental features of what has become the standard model of fish swimming. His first book on Mathematical Biofluiddynamics published by SIAM in 1975 covers a fuller account of both external and internal biofluid dynamics. His 1975 John von Neumann Lecture on Flagellar Hydrodynamics represents his significant work in mathematical biofluid dynamics of microorganisms with flagella and related organisms. He also developed mathematical methods required for the study of flagellar hydrodynamics. In particular, for flagellar locomotion, he developed a new viscous slender body theory which is superior to the classical dissipative force theory where Lighthill replaced a narrow long cylindrical flalgellum beating with a large wavelength by a distribution of Stokeslets and dipoles at its center-line whose strengths are calculated by means of integral equation representing the no-slip criterion on the cylindrical surface.

Lighthill also initiated a new field of internal biofluid dynamics and published several papers including two major papers on "Energy Flow in the Cochlea," and "Acoustic Streaming in the Ear Itself." The latter paper deals with biomechanics of cochlear traveling waves and with estimation of the magnitude of any mean streaming motions excited by acoustic signals after conversion into cochlear traveling waves. In particular, he demonstrated that the ability of the hair cells of the cochlea to sense different frequencies of sound according to their distance from the entrance could be related to a phenomenon of critical layer absorption of the elasto-acoustic waves generated in that organ.

In his famous paper on "The Recently Recognized Failure of Predictability in Newtonian Dynamics" which appeared in the Proceedings of the Royal Society of London in 1986, Sir James Lighthill vigorously argued against the belief that dynamical systems 
governed by Newton's laws of motion do not necessarily exhibit the predictability property. As a firm believer of Newtonian dynamics, Sir James' statement of public apology is an enlightenment to read: "Here I have to pause, and to speak once again on behalf of the broad global fraternity of practitioners of mechanics. We are all deeply conscious today that the enthusiasm of our forebears for the marvelous achievements of Newtonian mechanics led them to make generalizations in this area of predictability which, indeed, we may have generally tended to believe before 1960, but which we now recognize were false. We collectively wish to apologize for having misled the general educated public by spreading ideas about the determinism of systems satisfying Newton's laws of motion that, after 1960, were to be proved incorrect. In this lecture, I am trying to make belated amends by explaining both the very different picture that we now discern, and the reasons for it having been uncovered so late."

In another place of the same paper, he conclusively established the fact that: "My lecture, however, was about the mathematical properties of systems assumed to obey exactly the laws of Newtonian dynamics. The behaviour of such systems had long been thought to be completely predictable but is now known, for a certain proportion of such systems, to be 'chaotic' in a well defined sense.

There is nothing 'inexplicable' about this chaotic behaviour, a behaviour which (in fact) the mathematical analysis has fully explained, demonstrated and characterized. These mathematical theorems proved about the precise consequences of assuming Newtonian dynamical laws have the same immutability as all the 2000 year-old geometrical theorems about the consequences of assuming Euclid's axioms."

A thorough appreciation of Newton's great achievement was shown by Sir James in his following comments: "I feel fully justified, therefore, in repeating that systems subject to the laws of Newtonian dynamics include a substantial proportion of systems that are chaotic; and that, for these latter systems, there is no predictability beyond a finite predictability horizon. We are able to come to this conclusion without ever having to mention quantum mechanics or Heisenberg's uncertainty principle. A fundamental uncertainty about the future is there, indeed, even on the supposedly solid basis of the good old laws of motion of Newton, which effectively are the laws of motion satisfied by all macroscopic systems."

4. Concluding remarks. It is hoped that enough has been discussed to give some definite impression of Sir James Lighthill's professional life and career as well as of the range, power and depth of his great applied mathematical work in different areas of modern fluid dynamics. Sir James' whole career was totally dedicated to the pursuit of fundamental scientific discovery and dissemination of knowledge of diverse areas of fluid mechanics and applied mathematics. He was one of the most brilliant and influential fluid dynamists of this century. His research and publications have stimulated enormous interest among researchers in the academic as well as industrial community for further exploration and development of challenging problems in the fields of aerodynamics, aeroacoustics, waves in fluids, boundary layer theory, biofluid dynamics, atmospheric and ocean sciences. As a lifelong devotee of fluid dynamics, he expressed his genuine excitement for the subject in his 1962 lecture at the Fluid Dynamics Division of the American Physical Society by declaring: "It needs 
categorically to be reaffirmed that the continuum mechanics of a fluid innocent of electric field has as vital and exciting present and future as any other branch of physical science." As editor of Collected Papers of Sir James Lighthill, M. Yousuff Hussaini states the following in his General Introduction: "For more than half a century, his contributions spanned the fields of aeronautics, astrophysics, atmospheric and oceanographic sciences, and biofluid dynamics. But Lighthill is distinguished not just by the diversity and excellence of his technical contributions; his unique vision and perspicacity are evident in the groundbreaking, original work in every field he touched. In certain areas of aerodynamics and biofluid dynamics - such as wave propagation, aeroacoustics, and animal motion-his first articles were seminal and remain virtually the last word on the subject."

Sir James Lighthill was undoubtedly one of the most brilliant and influential fluid dynamists of the twentieth century. He revolutionized applied mathematics with his remarkable contributions to modern fluid dynamics. There is no doubt at all about Lighthill's profound and everlasting impact on mathematical sciences and the scientific community of the world. His lifelong concern for quality mathematics instruction at national and international levels, and for inevitable loss by natural hazards reveals the unique character of this man. He will be remembered forever not only for his great scientific achievements, but also his unique contribution to the welfare of the human race. In many ways, Sir James was the epitome of the applied mathematical community.

\section{REFERENCES}

[1] M. J. Lighthill, Two-dimensional supersonic aerofoil theory, Rep. and Memoranda, no. 1929, Ministry of Aircraft Production [London], Aeronaut. Res. Committee, 1944. MR 7,94a. Zbl 061.45801.

[2] _ On sound generated aerodynamically. I. General theory, Proc. Roy. Soc. London Ser. A 211 (1952), 564-587. MR 13,879i. Zbl 049.25905.

[3] _ - On sound generated aerodynamically. II. Turbulence as a source of sound, Proc. Roy. Soc. London, Ser. A 222 (1954), 1-32. MR 15,837e. Zbl 055.19109.

[4] T. B. Benjamin and M. J. Lighthill, On conoidal waves and bores, Proc. Roy. Soc. London Ser. A 224 (1954), 448-460. MR 17,911f. Zbl 055.45605.

[5] M. J. Lighthill, Studies on magnetohydrodynamic waves and other anisotropic wave motions, Philos. Trans. Roy. Soc. London Ser. A 252 (1960), 397-430. MR 26\#5844. Zbl 097.20806.

[6] _ Group velocity, J. Inst. Math. Appl. 1 (1965), 1-28. MR 32\#1930. Zbl 216.52201.

[7] _ A discussion on nonlinear theory of waves propagation in dispersive systems, Proc. Roy. Soc. London Ser. A 299 (1967), 1-145.

[8] Contributions to the theory of waves in nonlinear dispersive systems, J. Inst. Maths Appl. 1 (1965), 269-306.

[9] _ Some special cases treated by the Whitham theory, Proc. Roy. Soc. London Ser. A 299 (1967), 38-73.

[10] _ On waves generated in dispersive systems by travelling forcing effects, with applications to the dynamics of rotating fluids, J. Fluid Mech. 27 (1967), 725-752. Zbl 148.22804.

[11] _ Waves in fluids, Cambridge University Press, Cambridge, New York, 1978. MR 84g:76001a. Zbl 375.76001.

[12] _ Waves and hydrodynamic loading, Proc. 2nd Int. Conf. on Behavior of Offshore Structures, vol. 1, 1979, pp. 1-40. 
[13] _ Dynamic response of the Indian Ocean to the onset of the Southwest Monsoon, Philos. Trans. Roy. Soc. London Ser. A 265 (1969), 45-92.

[14] _ Mathematical biofluiddynamics, Regional Conference Series in Applied Mathematics, no. 17, Society for Industrial and Applied Mathematics, Philadelphia, Pa., 1975, Based on the lecture course delivered to the Mathematical Biofluiddynamics Research Conference of the National Science Foundation held from July 16-20, 1973, at Rensselaer Polytechnic Institute. MR 57 9113. Zbl 312.76076.

[15] _ The recently recognized failure of predictability in Newtonian dynamics, Proc. Roy. Soc. London Ser. A 407 (1986), 35-50. Zbl 608.70005.

[16] _ , The art of teaching the art of applying mathematics, Math. Gaz. 55 (1971), no. 393, 249-270. MR 589952.

[17] _ Presidential Address, Developments in Mathematical Education (A. G Howson, ed.), Cambridge University Press, 1973.

[18] M. Y. Hussaini, Collected Papers of Sir James Lighthill, vol. I-IV, Oxford University Press, Oxford, 1997.

[19] G. B. Whitham, Linear and nonlinear waves, Pure and Applied Mathematics. A WileyInterscience Series of Texts, Monographs, and Tracts, John Wiley \& Sons, New York, 1974. MR 58 3905. Zbl 373.76001.

[20] _ Non-linear dispersion of water waves, J. Fluid Mech. 27 (1967), 399-412. MR 34\#8711. Zbl 146.23702.

[21] _ Variational methods and applications to water waves, Proc. Roy. Soc. London Ser. A 299 (1967), 6-25. Zbl 163.21104.

[22] T. B. Benjamin and J. E. Feir, The disintegration of wave trains on deep water. I: Theory, J. Fluid Mech. 27 (1967), 417-430. Zbl 144.47101.

[23] T. B. Benjamin, Instability of periodic wavetrains in nonlinear dispersive systems, Proc. Roy. Soc. London Ser. A 299 (1967), 59-75.

[24] T. B. Benjamin and J. E. Feir, The disintegration of wavetrains on deep water, J. Fluid Mech. 27 (1967), 417-437, Part 2, Experiment.

[25] J. R. Morison, M. P. O'Brien, and J. W. Johnson, The force exerted by surface wave on piles, Petroleum Transactions 189 (1950), 149-154.

[26] L. Debnath, Nonlinear water waves, Academic Press, Inc., Boston, 1994. MR 95c:76011. Zbl 793.76001.

[27] L. Debnath (ed.), Nonlinear waves, Cambridge Monographs on Mechanics and Applied Mathematics, Cambridge University Press, Cambridge, 1983. MR 85d:76001. Zbl 536.00016.

[28] L. Debnath and M. Rahman, A theory of nonlinear wave loading on offshore structures, Internat. J. Math. Math. Sci. 4 (1981), no. 3, 589-613. MR 82h:76010. Zbl 468.76024.

[29] L. Debnath, Nonlinear partial differential equations for scientists and engineers, Birkhäuser Boston, Inc., Boston, 1997. CMP 97 14. Zbl 892.35001.

[30] L. Debnath (ed.), Advances in nonlinear waves. Vol. I, Research Notes in Mathematics, vol. 95, Pitman Advanced Publishing Program, Boston, London, Melbourne, 1984. MR 87a:76002a. Zbl 535.00028.

[31] _ Advances in nonlinear waves. Vol. II, Research Notes in Mathematics, vol. 111, Pitman Advanced Publishing Program, Boston, London, Melbourne, 1985. MR 87a:76002b. Zbl 563.00022.

[32] L. Debnath (ed.), Nonlinear Dispersive Wave Systems, World Scientific Publishing, Singapore, 1992. MR 93f:00015.

Debnath: Department of Mathematics, University of Central Florida, OrLando, FL 32816, USA 


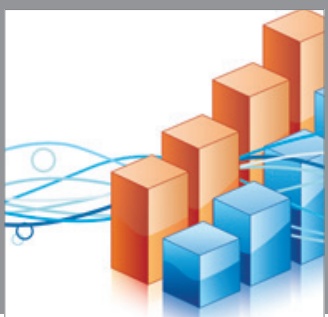

Advances in

Operations Research

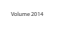

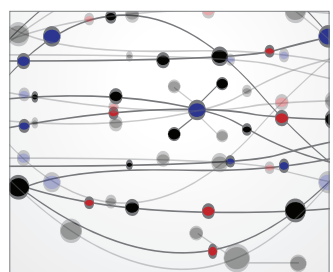

\section{The Scientific} World Journal
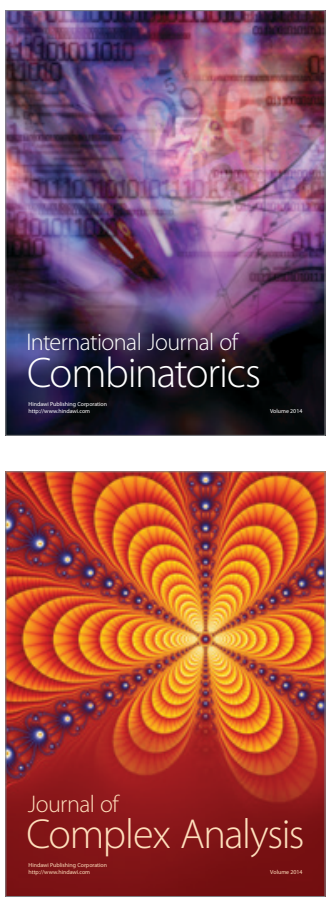

International Journal of

Mathematics and

Mathematical

Sciences
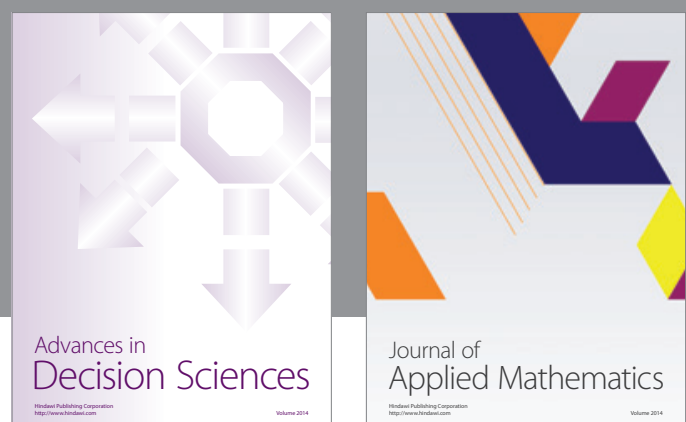

Journal of

Applied Mathematics
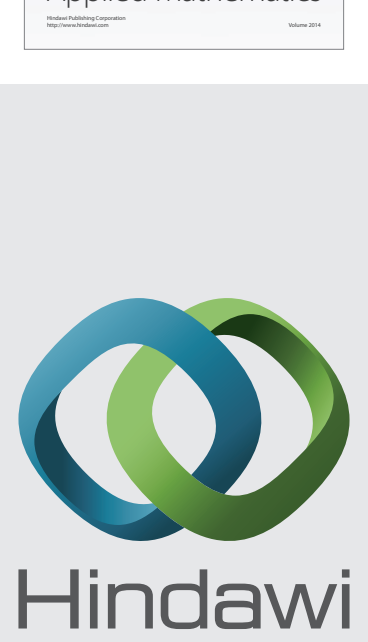

Submit your manuscripts at http://www.hindawi.com
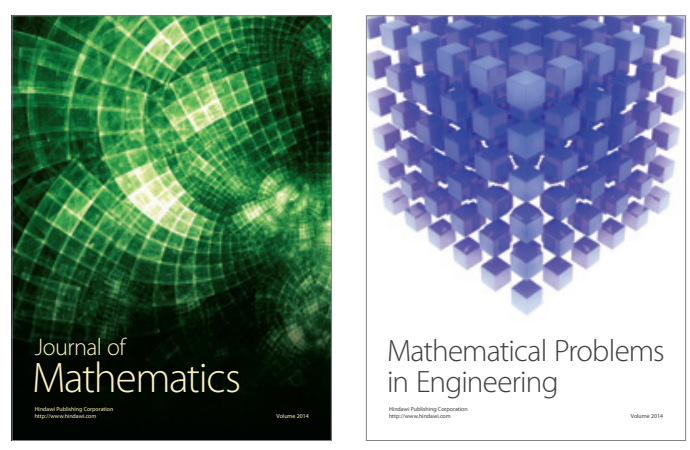

Mathematical Problems in Engineering
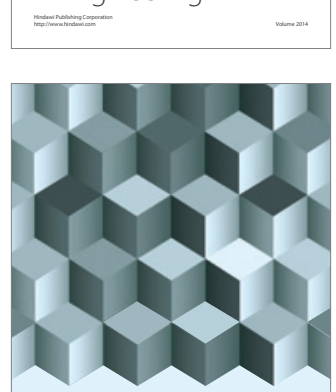

Journal of

Function Spaces
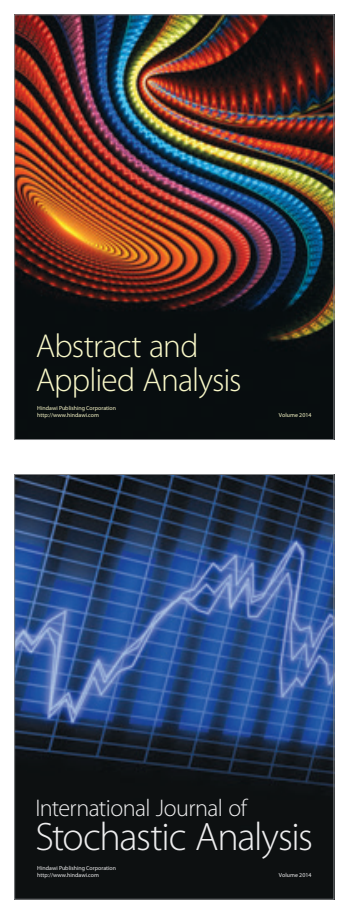

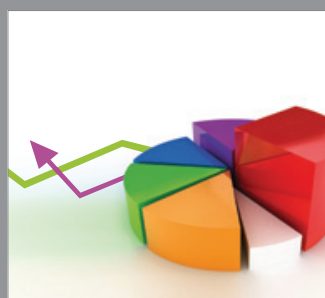

ournal of

Probability and Statistics

Promensencen
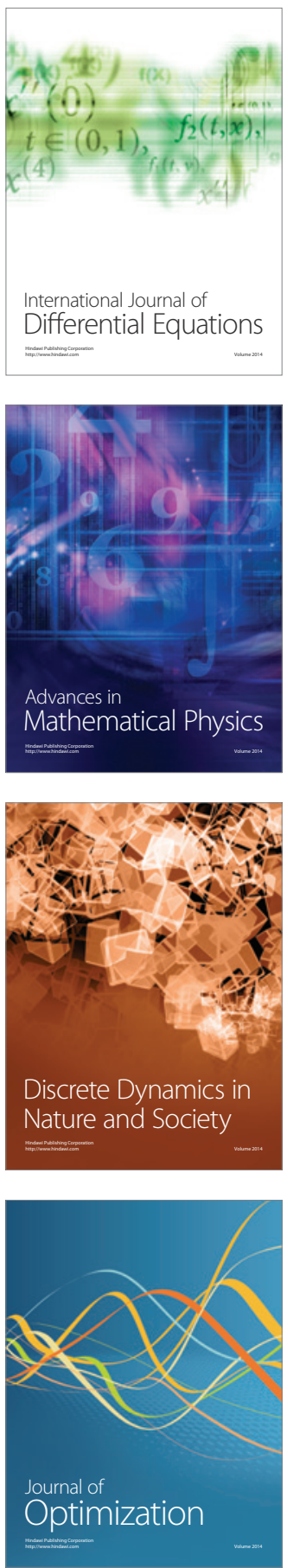